\title{
Conformational and Phase Transitions in DNA-Photosensitive Surfactant Solutions: Experiment and Modeling
}

\author{
N. Kasyanenko, ${ }^{1}$ L. Lysyakova, ${ }^{1,2}$ R. Ramazanov, ${ }^{1}$ A. Nesterenko, ${ }^{3,4}$ I. Yaroshevich, ${ }^{3}$ E. Titov, ${ }^{1}$ \\ G. Alexeev, ${ }^{1}$ A. Lezov, ${ }^{1}$ I. Unksov ${ }^{1}$ \\ ${ }^{1}$ Faculty of Physics, Department of Molecular Biophysics, Saint Petersburg State University, Petrodvorets, Ulyanovskaya str. \\ 1, 198504 St. Petersburg, Russia \\ ${ }^{2}$ Experimental Physics, Institute of Physics and Astronomy, University of Potsdam, Karl-Liebknecht-str. 24/25, 14469 \\ Potsdam-Golm, Germany \\ ${ }^{3}$ Faculty of Biology, Department of Biophysics, Lomonosov Moscow State University, Leninskie Gory 1/12, 119991 Moscow, Russia \\ ${ }^{4}$ A.N. Belozersky Institute of Physico-Chemical Biology, Lomonosov Moscow State University, Leninskie Gory 1/40, 119991 \\ Moscow, Russia
}

Received 11 July 2014; accepted 7 October 2014

Published online 10 October 2014 in Wiley Online Library (wileyonlinelibrary.com). DOI 10.1002/bip.22575

\section{ABSTRACT: \\ DNA binding to trans- and cis-isomers of azobenzene con- taining cationic surfactant in $5 \mathrm{mM} \mathrm{NaCl}$ solution was investigated by the methods of dynamic light scattering (DLS), low-gradient viscometry (LGV), atomic force microscopy (AFM), cincular dichroism (CD), gel electro- phoresis (GE), flow birefringence (FB), UV-Vis spectropho- tometry. Light-responsive conformational transitions of DNA in complex with photosensitive surfactant, changes in DNA optical anisotnpy and persistent length, phase transi- tion of DNA into nanoparticles induced by high surfactant concentration, as well as transformation of surfactant con- formation under its binding to macromolecule were stud- ied. Computer simulations of micelles formation for cis- and trans-isomers of azbenzene containing surfactant, as well as DNA-surfactant interaction, were carried out. Phase diagnam for DNA-surfactant solutions was designed. The}

Additiond Supporting Information may be found in the online verion of this artide.

Correpondence to: Nina Kasyanenko, o mail: nkasyanenlowimail.ru Contract gant sponeor: RFBR, G.REC, DAAD

Contract gant number: B3.03.01192 a

Contract gant sponoor: Saint Petersburg State University

Contract gant numbers: 11.38.644.2013, 11.50 1589.2013, 11.38.267.2014

Q 2014 Wiley Periodicals, Inc possibility to reverse the DNA packaging induced by surfactant binding with the dilution and light irradiation was shown. 2014 Wiley Periodicals, Inc. Biopolymers 103: 109-122, 2015.

Keywords DNA-surfactant complexes; light-induced DNA de-compaction; phase diagram; DNA volume and persistent length

This article was originally published online as an accepted preprint. The "Published Online" date corresponds to the preprint version. You can request a copy of any preprints from the past two calendar years by emailing the Biopolymers editorial office at biopolymers@wiley.com.

\section{INTRODUCTION}

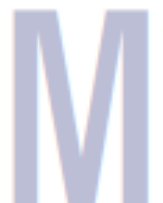

anipulation with high molecular DNA and investigation of its conformational possibilities after the addition of different compounds into the solution not only provide new knowledge about the structure and molecular basics of functions of the main biopolymer, but also allow to find a new approad for the construction of nanosystems having peculiar properties. Indeed, due to the unique charge density, hain rigidity, and double stranded structure of nucleic acid with hydrophobic bases inside the helix and hydrophilic sugar-phosphate carcass with high negative charge outside 